\title{
Continuous Separation of Model Proteins by Free- Flow Field Step Electrophoresis and its Electrokinetic Basis
}

Sunil Nath ( $\nabla$ sunil_nath_iit@yahoo.com )

Indian Institute of Technology Delhi

Research

Keywords: Bioseparation, Electrophoresis, Free buffer, Continuous, Preparative separation, Throughput, Concentration, Model proteins, Field step, Electrical conductivity

Posted Date: July 14th, 2021

DOI: https://doi.org/10.21203/rs.3.rs-702355/v1

License: (c) (1) This work is licensed under a Creative Commons Attribution 4.0 International License.

Read Full License 


\section{Continuous separation of model proteins by free-flow field step}

\section{electrophoresis and its electrokinetic basis}

Sunil Nath*

Department of Biochemical Engineering and Biotechnology, Indian Institute of Technology Delhi, Hauz Khas, New Delhi 110016, India

*E-mail: sunath@iitd.ac.in; sunil_nath_iit@yahoo.com

\section{Abstract}

Continuous separation of cells, cell membranes, and proteins by electrophoretic techniques such as free-flow zone electrophoresis (FFZE) has a rich history since its introduction sixty years ago by Hannig. However, the results of FFZE, capillary zone electrophoresis, and other similar electrophoretic techniques are limited to analytical scale separations that are not readily extendable to the preparative scale. Moreover, a five- to ten-fold dilution of samples by buffer is common in separations by these techniques. Free-flow field step electrophoresis (FFFSE) is an electrophoretic technique that appears to be suitable for continuous simultaneous preparative separation and concentration and therefore 
has the power to overcome the above limitations. Here we apply FFFSE to a simple system of model proteins to show proof-of-concept of the technique. A continuous, preparative-scale separation of myoglobin from BSA with a throughput of $20 \mathrm{mg} / \mathrm{h}$ and a yield of $>98 \%$ is shown to be successfully obtained using single-step FFFSE. Most important, it is shown that the preparative FFFSE experiment can be rationally designed, and the results predicted theoretically by use of electrokinetic data recorded in a simple analytical-scale FFZE experiment. This is the first paper to present a theory of separation in FFFSE. The separation is continuous, single-step, and environmental-friendly as no adjuvants are used, with no need for regeneration of components. The electrokinetic basis of the separation appears to be of a general nature. In further research we are testing the limits of the technique, by exploring its extension to more complex systems, and to higher preparative-scale throughputs.

Keywords: Bioseparation; Electrophoresis; Free buffer; Continuous; Preparative separation; Throughput; Concentration; Model proteins; Field step; Electrical conductivity 


\section{Introduction}

Electrophoresis in free buffer offers an attractive approach for carrying out bioseparation in a continuous mode [1]. Among an array of free-flow electrophoretic techniques [2,3], free-flow zone electrophoresis (FFZE) is the best known, and its field of application is very broad. It has been successfully used for the separation of cells, cell debris, populations of organelles as well as protein mixtures [1-7]. A merit of the FFZE process, which ought to be stressed, is its ability to maintain biological activity of samples given that the separation is achieved in free fluid in the absence of any solid support or gel. The technique has also been shown to be very useful for complex mixtures such as fermentation crude extracts without the necessity of any additional purification step $[6,8,9]$.

However, like all techniques, the FFZE technique presents certain limitations. Most of the documented results of FFZE are applicable to analytical scale separations [10] and cannot be easily extended to the preparative scale. Moreover, at least a five-fold dilution factor of samples by buffer is commonly observed in FFZE, and hence the technique is not ideally suitable for continuous simultaneous electrophoretic separation and concentration [6,9]. Free-flow field 
step electrophoresis (FFFSE) appeared to be a promising alternative technique to achieve this aim, although free-flow isoelectric focusing, moving reaction boundary electrophoresis and other predictive techniques have been pioneered in the literature [11-19], notably by Ivory and colleagues $[3,15-18]$.

A literature search revealed that other than the continuous separation of the dye rhodamine B from fluorescein [2], no applications to preparative protein separation had been documented by the FFFSE technique. Turgeon and Bowser [11] also reported that no further data using FFFSE was available. The situation has remained virtually unchanged since then, though many interesting approaches to the general problem of protein complexation and separation have evolved, and new media and approaches have been tried out [20-25]. It is the purpose of this work to apply FFFSE to a simple system of model proteins, develop a theory of separation, and highlight its electrokinetic basis. However, it is not our aim to provide a catalog of successful applications. Above all, it is shown that a simultaneous separation and concentration process in FFFSE can be successfully designed and predicted using electrophoretic mobility data obtained from a simple FFZE experiment. The underlying principle of the FFFSE process is outlined in Section 2. 


\section{Principle of FFFSE}

As mentioned in Section 1, analytical techniques such as FFZE are beset by a large number of difficulties. In these techniques, the sample is diluted by buffer, and a number of factors lead to band/zone broadening and negatively influence the quality of the separation. Primary factors of band/zone broadening and loss of separation efficiency arise from disturbances arising from the parabolic flow profile and from electroosmosis [2]. Although some innovative ways to mitigate the effects of these disturbances and optimizing FFZE separations have been described in the literature [6], the fact remains that most FFZE applications are still limited by resolution.

The practical implementation of FFZE leads to high dispersion of samples and therefore to poor separation performance, as discussed above. A major cause of these deleterious effects in FFZE is the use of buffer with constant physical properties throughout the width of the separation channel. These difficulties are circumvented in FFFSE by changing the assumption of constant physical properties of the buffer in the separation chamber (Fig. 1). In particular, differences in electrical conductivity, $\kappa$ of media within the separation chamber (Fig. 1) permits rapid migration and separation of analytes in the low $\kappa$ regions 
in FFFSE (Fig. 2). It also serves to focus and concentrate the charged species as they approach the boundaries of the high $\kappa$ regions, since the species now experience a small value of the electrical field strength, $E$ given that $E \propto \kappa^{-1}$ (Figs. 1, 2). Thus at high sample flow rates, band broadening of analytes is minimized in FFFSE, and simultaneous separation and concentration can be achieved continuously on a preparative scale (Fig. 2).

In current practice, FFFSE uses high conductivity buffers only along the edges of the separation chamber. These create boundary walls at the two edges along which the electrophoretic mobility of the samples decrease, causing focusing of the bands (Fig. 2). This design requires some modifications for preparative separation of proteins by FFFSE, as discussed in subsequent Sections.

\section{Experimental}

\subsection{Electrophoretic apparatus}

The rectangular plexiglass electrophoretic apparatus used for the FFZE and FFFSE studies was assembled in-house, with dimensions of length $500 \mathrm{~mm}$, width $100 \mathrm{~mm}$ and thickness $0.5 \mathrm{~mm}$. The constant gap of $0.5 \mathrm{~mm}$ between the plexiglass front plate and the mirrored copper back plate was maintained by a 
rigid polypropylene spacer. Two electrode compartments were located on each side of the separation chamber and were isolated from the latter using cellulose acetate membranes. The outlet of the separation chamber consisted of 90 collection ports each $1.11 \mathrm{~mm}$ along the width of the chamber.

Buffer was fed continuously at the top of the chamber. The sample was also injected continuously at the top from a needle with a diameter of $1 \mathrm{~mm}$. The electrical field was applied in a direction perpendicular to the buffer flow with the help of two platinum electrodes and a power supply.

Convection currents arising from heat gradients in the separation chamber were prevented by the use of cooling systems. High conductivity buffer circulation around the electrodes removed the heat generated due to the Joule effect, and the temperature was controlled at $15 \pm 0.5^{\circ} \mathrm{C}$ in the chamber. All electrophoretic migrations were measured from the position where the sample eluted at zero electric field. 


\subsection{Analytical methods}

Separation results were evaluated from concentration measurements at the exit fraction ports of the chamber. Myoglobin was estimated from optical density measurements at $410 \mathrm{~nm}$, and the BSA from absorption at $280 \mathrm{~nm}$. The Soret absorption peak at $410 \mathrm{~nm}$ in the absorption spectrum of myoglobin is entirely due to the heme, and is a signature of all heme proteins. Use of a microcolorimetric Bradford procedure and bicinchoninic acid BCA assay led to similar estimation results. All protein samples were dissolved in Tris-acetate chamber buffer. Electrical potentials between 0.5 and $1.5 \mathrm{kV}$, and buffer residence times between $5 \mathrm{~min}$ and $2 \mathrm{~min}$ were tested. This corresponded to a sample flow rate for the mixture of model proteins of $1.5 \mathrm{~mL} / \mathrm{h}$ to $6.2 \mathrm{~mL} / \mathrm{h}$ for injection in a single fraction. The FFZE was carried out at a constant chamber buffer electrical conductivity of $1.5 \mathrm{mS} / \mathrm{cm}$. For the FFFSE experiments, the sample of the protein mixture containing each components at a final concentration of $1 \mathrm{mg} / \mathrm{mL}$ each was injected in a broad zone between fractions 20 and 30 in $0.5 \mathrm{mS} / \mathrm{cm}$ buffer, and two steps in electrical conductivity were employed. The first was from 0.5 to $1.5 \mathrm{mS} / \mathrm{cm}$ with the second from 1.5 to 9.0 mS/cm (Fig. 3). 


\section{Results and Discussion}

\subsection{Design of separation process by FFFSE}

Several preliminary experiments conducted on model proteins revealed that it was not possible to obtain a useful preparative-scale continuous separationcum-concentration with FFFSE if the corresponding continuous separation with FFZE (albeit with an approximately five-fold dilution) was unsuccessful. In the present context, these results implied that the electrophoretic mobility of the model proteins as determined in an analytical experiment were sufficiently different from each other (i.e. with a difference of $\sim 2 \times 10^{-5}-3 \times 10^{-5}$ $\mathrm{cm}^{2} /(\mathrm{Vs})$. These experiments also established that it was critical to select experimental conditions such that both/all the components migrated in the same direction during preparative FFFSE. In other words, the conventional design approach to the separation - in which the two components possess opposite net charge and migrate in opposite directions in the imposed electrical field (Fig. 2) - was not very satisfactory. A superior design was to employ a configuration with the electrical conductivity profile as shown in Fig. 3, i.e. one in which both species exhibited a net charge of the same sign and moved in the same direction in the field. 
Table 1 tabulates the electrophoretic mobility obtained from separate analytical electrophoresis experiments on the model proteins myoglobin and BSA. The sample of the individual protein was injected at fraction 27. A Tris acetate chamber buffer of $\mathrm{pH} 7.7$ with an electrical conductivity of $1.5 \mathrm{mS} / \mathrm{cm}$ was employed. The buffer residence time in the chamber measured 5 min and the electrical field strength was $130 \mathrm{~V} / \mathrm{cm}$, with a current of $0.3 \mathrm{~A}$ (Table 1).

Next an FFFSE experiment using two anodic conductivity steps from $0.5-1.5$ $\mathrm{mS} / \mathrm{cm}$ and $1.5-9.0 \mathrm{mS} / \mathrm{cm}$ in a Tris-acetate buffer of $\mathrm{pH} 7.7$ was designed (Fig. 3) for the mixture of myoglobin and BSA of $1 \mathrm{mg} / \mathrm{mL}$ final concentration. The sample was injected continuously in the lowest conductivity zone of 0.5 $\mathrm{mS} / \mathrm{cm}$ between fractions 20 and 30 at a consolidated flow rate of approximately $20 \mathrm{ml} / \mathrm{h}$ using a specifically tailored polypropylene spacer to direct the flow. This flow rate corresponded to $2 \mathrm{ml} / \mathrm{h}$ per fraction. A voltage of $1.3 \mathrm{kV}$ was applied across the chamber. The results of this FFFSE experiment are shown in Fig. 4. An approximately four-fold concentration of myoglobin and BSA along with their simultaneous separation was obtained, with a throughput of $20 \mathrm{mg} / \mathrm{h}$ was obtained using FFFSE with a yield >98\% (Fig. 4). 
In order to rationalize the FFFSE separation shown in Fig. 4, an analytical FFZE experiment on more than a dozen proteins was conducted and their electrophoretic mobilities determined, as shown in Fig. 5. The electrophoretic mobility of the subset of myoglobin and BSA proteins obtained from this analytical experiment is tabulated in Table 1. From this data, the migration distance of myoglobin and BSA was calculated based on Eq. (1) [6], and this was used to determined the position of the conductivity steps in preparative FFFSE. The equation and model calculations are given by:

$$
d=\frac{2}{3} \frac{u \tau I}{A \kappa}
$$

where $d$ measures the distance of migration in the electrical field, $u$ is the electrophoretic mobility of the protein, $\tau$ the residence time in the electrophoresis chamber, $I$ the current, $A$ the cross-sectional area of the chamber, and $\kappa$ is the conductivity of the medium. The factor $\frac{2}{3}$ accounts for the correction due to the parabolic profile of the Poiseuille flow of buffer and sample in the electrophoresis chamber [6,9].

Applying Eq. (1) with the parameters in Table 1, we find for BSA 


$$
d=\frac{2 \times 3.4 \times 10^{-5} \mathrm{~cm}^{2} /(\mathrm{Vs}) \times(5 \times 60) \mathrm{s} \times 0.3 \mathrm{~A}}{3 \times(10 \times 0.05) \mathrm{cm}^{2} \times 1.5 \times 10^{-3} \mathrm{~S} / \mathrm{cm}}
$$

Or

$$
d=2.7 \mathrm{~cm}
$$

The number of fractions migrated, $n$ by BSA is given by

$$
n=\frac{2.7 \mathrm{~cm}}{(10 / 90) \mathrm{cm}}
$$

which works out to $n=24$ fractions. Hence if the BSA is injected at fraction number 27 in a buffer of $\kappa=1.5 \mathrm{mS} / \mathrm{cm}$, then its peak will elute at fraction number 51. Similar calculations based on the data of table 1 show that the peak of a sample of myoglobin injected at fraction 27 will elute at fraction 30 in buffer of the same conductivity. 
The above discussion has shown that application of the governing electrokinetic equation of FFZE [6,9] together with the values of electrophoretic mobilities of the components in the separation process shown in Table 1 yielded a migration distance, $d$ of $2.7 \mathrm{~cm}$ for BSA and $0.32 \mathrm{~cm}$ for myoglobin. From the physical arrangement of the output tubes (Section 3), this implies that the peak of the myoglobin migrated 3 fractions while the peak of the BSA moved 24 fractions during FFZE. Since the injection in the FFZE experiment was at fraction 27, the peak of myoglobin should elute at fraction number 30, and the peak of BSA should elute at fraction number 51 in chamber buffer of electrical conductivity $1.5 \mathrm{mS} / \mathrm{cm}$.

The above calculation involved only a single point injection (at fraction number 27), and migration in a chamber buffer characterized by a single value of the electrical conductivity, $\kappa$. Hence the calculation was simple and straightforward. However, in the corresponding FFFSE experiment, the injection occurs as a broad zone that covers multiple points (fraction numbers 20 to 30, i.e. about 10 fractions in the experiment shown in Fig. 4). Moreover, the values of $\kappa$ change with space along the width of the FFFSE separation chamber and vary between 0.5 to $1.5 \mathrm{mS} / \mathrm{cm}$ and from 1.5 to $9.0 \mathrm{mS} / \mathrm{cm}$ due to the two anodic conductivity steps imposed during the experiment (Figs. 3, 4). This is due to the fact that $\kappa=0.5 \mathrm{mS} / \mathrm{cm}$ was imposed between fraction numbers 20 and $30, \kappa=$ 
$1.5 \mathrm{mS} / \mathrm{cm}$ between fraction numbers 30 and 36 , and $\kappa=9.0 \mathrm{mS} / \mathrm{cm}$ between fractions 36 and 40 in the FFFSE experiment (Figs. 3, 4). For the conditions of the FFFSE experiment of Fig. 4, the zone electrophoresis equation [6] applicable to predict the elution fractions in FFFSE therefore adopts the form,

$d_{i} \kappa_{i}=d_{j} \kappa_{j}$

where $d_{i}, d_{j}$ represent the distance migrated in the electrophoretic experiment in any two zones $i$ and $j$ of the FFFSE chamber with varying conductivity properties, $\kappa_{i}, \kappa_{j}$.

Further, we have to take into consideration both the left edge (at fraction number 20) and the right edge (at fraction number 30) of the injection in the FFFSE experiment and then calculate the actual number of fractions moved during FFFSE using Eq. (5) to account for the spatial variation of $\kappa$ in the separation chamber. This calculation has been performed for the peak and left and right edges, and the results have been tabulated in Table 2 . 
A sample calculation for BSA will help to elucidate the calculation procedure for the spread of the distribution of the BSA concentration. Consider for example the right front of the BSA. The right edge of the injection at fraction 30 will move a number of fractions equal to $6+\frac{(24-6)}{6}$, i.e. it will elute at fraction number $(30+9)=39$ and focus, since it initially encounters a conductivity $\kappa$ $=1.5 \mathrm{mS} / \mathrm{cm}$, the same as that used in the FFZE experiment of Fig. 5, followed by a zone of conductivity that is six-fold higher. On the other hand, for the injection at fraction number 20, from Eq. (5), three fractions of $\kappa=1.5 \mathrm{mS} / \mathrm{cm}$ are taken up to move from fraction numbers 20 to 30 of $\kappa=0.5 \mathrm{mS} / \mathrm{cm}$ buffer, or nine fractions in the $\kappa=0.5 \mathrm{mS} / \mathrm{cm}$ buffer. Hence the BSA injection at fraction number 20 should move, from electrokinetic theory, a number of fractions equal to [9+6+2], i.e. 17 fractions, and it should therefore elute at fraction number 37 and focus (Table 2). The spread of the distributions calculated using Eq. (5) and tabulated in Table 2 are in good agreement with the observed distributions of the individual species (Fig. 4).

The results of Table 2 suggest that the reason for the high yield obtained in the FFFSE separation of Fig. 4 arises from the fact that the BSA migrates towards the anode and reaches the edge of the highest conductivity, $\kappa=9.0 \mathrm{mS} / \mathrm{cm}$ at fractions $36-40$, and thereby focuses at the second anodic conductivity step (Fig. 4). On the other hand, the lower electrophoretic mobility of myoglobin 
(Table 1) only lets it migrate to fraction 33, as results of similar calculations (Table 2) show that it distributes between fractions 29 and 33, and that it does not reach fraction 36 where $\kappa=9.0 \mathrm{mS} / \mathrm{cm}$. Thus the myoglobin fraction focuses at the first anodic conductivity step, as found in Fig. 4.

The above analysis leads to the thumb-rule that experimental conditions in FFFSE should be selected such that one component reaches the edge of the highest conductivity zone and focuses there upon experiencing a sudden reduction in the electrical field, while the other component only migrates within the low and the intermediate conductivity zone, and does not reach the edge of the highest conductivity zone (Table 2). Such spatial separation causes minimum protein-protein interactions and leads to maximum yield of separation. In principle, this separation strategy possesses an electrokinetic basis, and can be extended to multicomponent mixtures, with a larger number of conductivity steps.

Experiments are currently ongoing to extend the technique to other protein mixtures. The correlation of Fig. 5 includes 13 components, and pair-wise separation would require that a number of independent experiments equal to ${ }^{13} \mathrm{C}_{2}$, i.e., as many as 78 experiments be conducted! This is a very large number 
of experiments. Therefore, currently we are exploring the throughput limits of the FFFSE technique on an important sub-set of the samples, the biologically important separation of $D-$ and $L-$ dehydrogenases. The maximum throughput by such continuous, single-step electrophoresis techniques corresponds to a preparative scale separation of $\sim 5 \mathrm{~g} / \mathrm{h}$ of the dehydrogenases (Nath et al., in preparation). Hence the FFFSE technique has several applications in the field of bioseparation that have been relatively unexplored, and therefore we are only at the beginning of establishing the potential of this novel technique.

\section{Conclusion}

The present work describes a continuous, single-step procedure for preparative separation by free-flow field step electrophoresis by a new design approach of that employs multiple anodic electrical conductivity steps. The experiment is designed such that each component of the system focuses at a separate conductivity step. A continuous, preparative scale separation of model proteins myoglobin from BSA with a throughput of $20 \mathrm{mg} / \mathrm{h}$ could be successfully obtained with a yield of $>98 \%$ using the above methodology by FFFSE. Variables that need to be experimentally optimized include the electrical 
conductivity, $\kappa$ of the intermediate stage, the concentration of the protein mixture, the magnitude of the applied electrical field, and the operating $\mathrm{pH}$. The above results appear to be of a general nature and have the potential to be extended to other binary systems using the electrokinetic properties determined in Fig. 5, and also to multicomponent separations. This arises because the indices $i$ and $j$ in Eq. (5) can be assigned pairwise between any two components of a system, and the theory offers no constraint to such an assignment. The separation by FFFSE is shown to possess an electrokinetic basis. The migration of proteins, and thereby the separation, is shown to be predicted successfully using experimentally determined electrophoretic mobilities. The free-fluid nature of the separation ensures that no additional post-separation or regeneration steps are required, thereby making the process environmentally friendly. It can safely be applied and should prove to be valuable for a number of preparative separation processes in various engineering contexts. 


\section{Abbreviations}

BSA: Bovine serum albumin; FFFSE: Free-flow field step electrophoresis; FFZE: Free-flow zone electrophoresis

\section{Declarations}

Ethics approval and consent to participate

Not applicable

\section{Consent for publication}

Not applicable

\section{Availability of data and materials}

All are included in the article

\section{Competing interests}

The author declares no competing interests

\section{Funding}

No funding was received for this particular work

\section{Author contributions}

S.N. conceived the study, arranged materials, conducted the study, analyzed the data, and wrote the paper 


\section{Acknowledgements}

Not applicable 


\section{References}

[1] F.J. Agostino, S.N. Krylov, Advances in steady-state continuous-flow purification by small-scale free-flow electrophoresis, Trends Anal. Chem. $72(2015) 68-79$.

[2] D. Kohlheyer, J.C.T. Eijkel, A. van den Berg, R.B.M. Schasfoort, Miniaturizing free-flow electrophoresis - a critical review, Electrophoresis 29 (2008) 977-993.

[3] C.F. Ivory, The prospects for large-scale electrophoresis, Sep. Sci. Technol. 23 (1988) 875-912.

[4] G. Schmitz, A. Böttcher, H.G. Kahl, T. Brüning, Purification of monoclonal antibodies against the low density lipoprotein receptor by preparative isotachophoresis, J. Chromatogr. 432 (1988) 327-342.

[5] P.G. Righetti, E. Wenisch, A. Jungbauer, H. Katinger, M. Faupel, Preparative purification of human monoclonal antibody isoforms in a multicomponent electrolyser with immobiline membranes, J. Chromatogr. 500 (1990) 681-696.

[6] S. Nath, H. Schütte, H. Hustedt, W.-D. Deckwer, Application of continuous zone electrophoresis to preparative separation of proteins, Biotechnol. Bioeng. 42 (1993) 829-835. 
[7] L. Křiváncová, P. Boček, Continuous free-flow electrophoresis, Electrophoresis 19 (1998) 1064-1074.

[8] S. Hoffstetter-Kuhn, H. Wagner, Scale-up of free flow electrophoresis: I. Purification of alcohol dehydrogenase from a crude yeast extract by zone electrophoresis, Electrophoresis 11 (1990) 451-456.

[9] S. Nath, H. Schütte, H. Hustedt, W.-D. Deckwer, G. Weber, Separation of enzymes from microorganism crude extracts by free-flow zone electrophoresis, Biotechnol. Bioeng. 51 (1996) 15-22.

[10] K. Hannig, H. Wirth, R.K. Schindler, K. Spiegel, Free-flow electrophoresis: An analytical version for a rapid, quantitative determination of electrophoretic parameters, Hoppe-Seyler's Z. Physiol. Chem. 358 (1977) 753-763.

[11] R.T. Turgeon, M.T. Bowser, Micro free-flow electrophoresis: Theory and applications, Anal. Bioanal. Chem. 394 (2009) 187-198.

[12] M.L. Grant, D.A. Saville, Electrostatic interactions between a nonuniformly charged sphere and a charged surface, J. Coll. Interface Sci. $171(1995) 35-45$

[13] S. Nath, Correlation of migration behavior in free-flow zone electrophoresis and electrophoretic titration curve, Electrophoresis 11 (1990) 612-616. 
[14] M. Poggel, T. Melin, Free-flow zone electrophoresis: A novel approach and scale-up for preparative protein separation, Electrophoresis 22 (2001) $1008-1015$.

[15] C.F. Ivory, A brief review of alternative electrofocusing techniques, Sep. Sci. Technol. 35 (2000) 1777-1793.

[16] Z. Huang, C.F. Ivory, Digitally controlled electrophoretic focusing, Anal. Chem. 71 (1999) 1628-1632.

[17] C.F. Ivory, Preparative free-flow electrophoresis in a vortex-stabilized annulus, Electrophoresis 25 (2004) 360-374.

[18] B.M. Thome, C.F. Ivory, Increasing the scale of true moving bed electrophoretic separations using filtration to reduce solvent volumetric flows, J. Chromatogr. A 1138 (2007) 291-300.

[19] M. Aider, D. de Halleux, L. Bazinet, Potential of continuous electrophoresis in the bio-food industry, Trends Food Sci. Technol. 19 (2008) 351-362.

[20] K. Hannig, New aspects in preparative and analytical continuous freeflow cell electrophoresis, Electrophoresis 3 (1982) 235-243.

[21] B. Lammel, A method for continuous preparative electrophoresis using a supporting medium, Electrophoresis 2 (1981) 39-45. 
[22] S. Nath, Complexation behavior of proteins with polyelectrolytes, J. Chem. Tech. Biotechnol. 62 (1995) 295-300.

[23] S. Nath, Separation of enzymes from Candida boidinii crude extract by continuous flow zone electrophoresis, Electrophoresis 11 (1990) 937-941.

[24] L. Aljerf, F. Dehmchi, V.T. Pham, A.L. Choukaife, Polyurethane foam in a reliable method for electrophoretic separation of proteins, Am. Res. J. Chem. 3 (2019) 1-14.

[25] Y.H. Chow, A. Sahlini, H.-S. Nig, J.C.-W. Lan, Efficacy of alcohol/sugar aqueous biphasic system on partition of bovine serum albumin, Bioresour. Bioprocess. 8 (2021) 7. 


\section{Legends to the Figures}

Fig. 1. Principle of free-flow field step electrophoresis (FFFSE). Profile of electrical conductivity, $\kappa$ and electrical field strength, $\mathrm{E}$ in the standard FFFSE configuration.

Fig. 2. Operation of FFFSE. In the standard configuration, the two components in the mixture migrate in opposite directions and focus at the edges of the single electrical conductivity step.

Fig. 3. Modified configuration of FFFSE for separation of model proteins. The sample containing a mixture of proteins is injected in a broad zone between fractions 20 and 30 in the lowest electrical conductivity media, and two anodic conductivity steps are employed. The individual components of the mixture migrate very rapidly in the electrical field, and each component gets focused at the respective conductivity step immediately after fraction numbers 30 and 36 .

Fig. 4. Separation of model proteins by continuous FFFSE with two anodic conductivity steps. Experimental conditions: Tris acetate buffer, $\mathrm{pH} 7.7, \kappa=0.5$, 
$1.5,9.0 \mathrm{mS} / \mathrm{cm}$; buffer residence time $=5 \mathrm{~min}$, voltage $=1.3 \mathrm{kV}$. The sample of the mixture of model proteins of $1 \mathrm{mg} / \mathrm{mL}$ concentration each after mixing was injected as a broad zone between fraction numbers $20-30$. Bold line: Absorption at $410 \mathrm{~nm}$, Myoglobin. Dashed line: Absorption at $280 \mathrm{~nm}$, BSA. The profile of electrical conductivity, $\kappa$ and the conductivity discontinuities across the separation chamber were as follows: Fractions $20-30, \kappa=0.5$ $\mathrm{mS} / \mathrm{cm}$; fractions $30-36, \kappa=1.5 \mathrm{mS} / \mathrm{cm}$; fractions $36-40, \kappa=9.0 \mathrm{mS} / \mathrm{cm}$.

Fig. 5. Correlation determined to help establish an electrokinetic basis for separation in FFZE/FFFSE for various proteins $\left(\mathrm{R}^{2}=0.98\right)$. Tris acetate buffer, $\mathrm{pH}$ 7.7. The various proteins in ascending order of electrophoretic mobility are 1: myoglobin; 2: IgG; 3: formate dehydrogenase from Candida boidinii; 4: catalase from bovine liver (sample 1); 5: D-Hic dehydrogenase; 6: catalase from bovine liver (sample 2); 7: formaldehyde dehydrogenase from Candida boidinii; 8: bovine serum albumin (BSA); 9: ovalbumin from chicken egg; 10: ferritin from horse spleen; 11: $\beta$-galactosidase from Escherichia coli; 12: bovine thyroglobulin; 13: methanol oxidase from Candida boidinii; 14: L-Hic dehydrogenase. 


\section{Title of Tables}

Table 1. Electrophoretic mobility of model proteins myoglobin and BSA determined by analytical scale FFZE (Fig. 5).

Table 2. Prediction of electrophoretic migration of myoglobin and BSA in the continuous FFFSE experiment shown in Fig. 4 with an optimized intermediate $\kappa$ $=1.5 \mathrm{mS} / \mathrm{cm}$ and two anodic conductivity steps $(\kappa=0.5-1.5 \mathrm{mS} / \mathrm{cm}$ and $\kappa=$ $1.5-9.0 \mathrm{mS} / \mathrm{cm})$. The mixture was injected as a broad band from fraction numbers 10 to 20 in the $\kappa=0.5 \mathrm{mS} / \mathrm{cm}$ conductivity zone of the separation chamber. The spread of the distribution of outlet fraction numbers at which the myoglobin and BSA components elute from the chamber, after accounting for the width of the injection, is calculated using Eq. (1) and Eq. (5). Each fraction moved corresponds to a distance of electrophoretic migration of $0.11 \mathrm{~cm}$. 
Table 1. Electrophoretic mobility of model proteins myoglobin and BSA determined by analytical scale FFZE (Fig. 5).

\begin{tabular}{|l|l|}
\hline Protein & Electrophoretic mobility $\left(\mathrm{cm}^{2} /(\mathrm{Vs})\right.$ \\
\hline Myoglobin & $0.4 \times 10^{-5}$ \\
\hline BSA & $3.4 \times 10^{-5}$ \\
\hline
\end{tabular}


Table 2. Prediction of electrophoretic migration of myoglobin and BSA in the continuous FFFSE experiment shown in Fig. 4 with an optimized intermediate $\kappa$ $=1.5 \mathrm{mS} / \mathrm{cm}$ and two anodic conductivity steps $(\kappa=0.5-1.5 \mathrm{mS} / \mathrm{cm}$ and $\kappa=$ $1.5-9.0 \mathrm{mS} / \mathrm{cm})$. The mixture was injected as a broad band from fraction numbers 10 to 20 in the $\kappa=0.5 \mathrm{mS} / \mathrm{cm}$ conductivity zone of the separation chamber. The spread of the distribution of outlet fraction numbers at which the myoglobin and BSA components elute from the chamber, after accounting for the width of the injection, is calculated using Eq. (1) and Eq. (5). Each fraction moved corresponds to a distance of electrophoretic migration of $0.11 \mathrm{~cm}$.

\begin{tabular}{|c|c|c|c|}
\hline $\begin{array}{l}\kappa \\
(\mathrm{mS} / \mathrm{cm})\end{array}$ & $\begin{array}{l}\text { Fraction numbers } \\
(\kappa)\end{array}$ & $\begin{array}{l}\text { Spread of elution fraction } \\
\text { numbers (Myoglobin) }\end{array}$ & $\begin{array}{l}\text { Spread of elution } \\
\text { fraction numbers (BSA) }\end{array}$ \\
\hline 0.5 & $20-30$ & Sample injection & Sample injection \\
\hline 1.5 & $30-36$ & $29-33$ & $38-40$ \\
\hline 9.0 & $36-40$ & Does not reach fraction 36 & \\
& & Focuses at first $\kappa$ step & Focuses at second $\kappa$ step \\
\hline
\end{tabular}


Figures
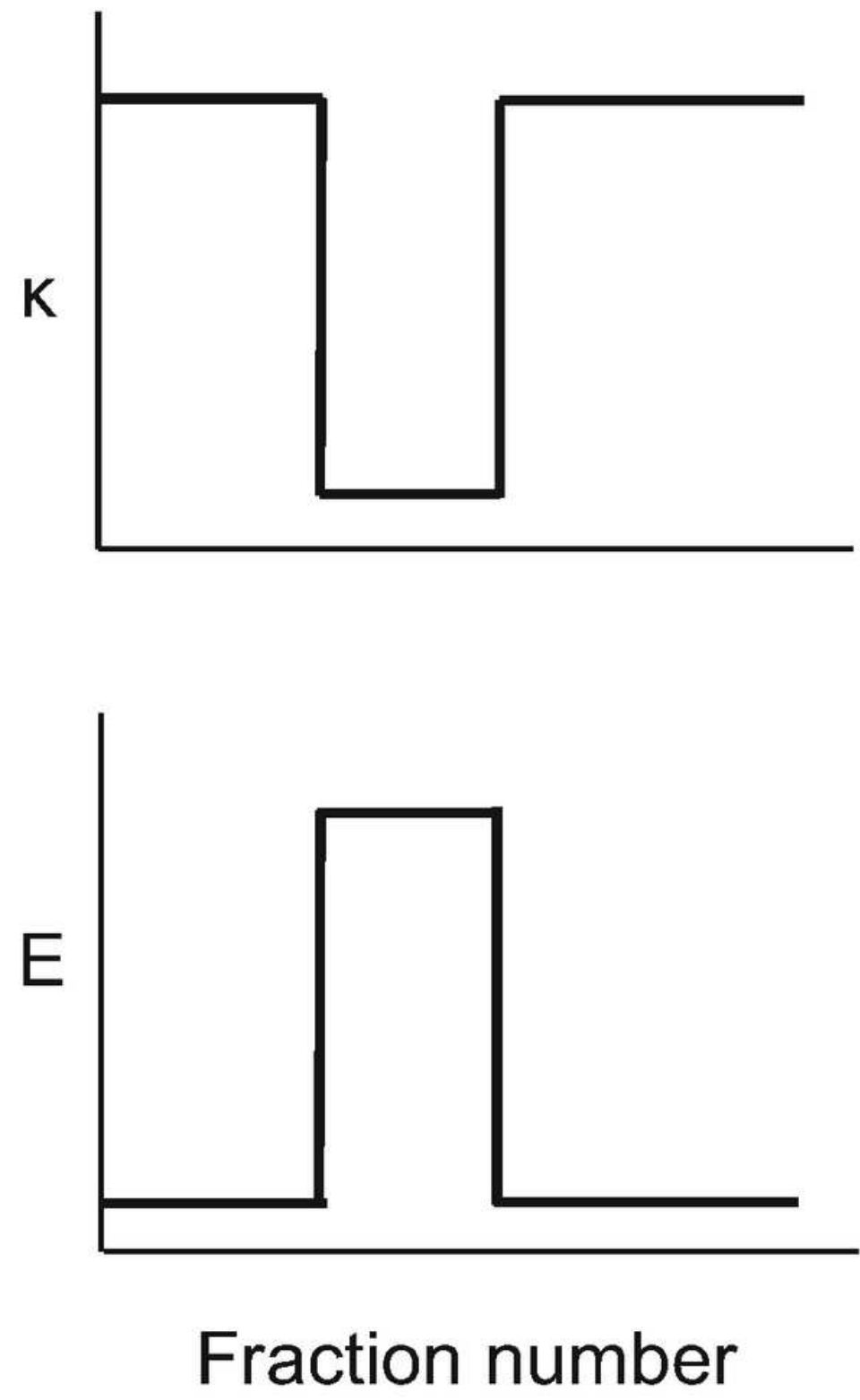

Figure 1

Principle of free-flow field step electrophoresis (FFFSE). Profile of electrical conductivity, $\mathrm{k}$ and electrical field strength, $E$ in the standard FFFSE configuration. 


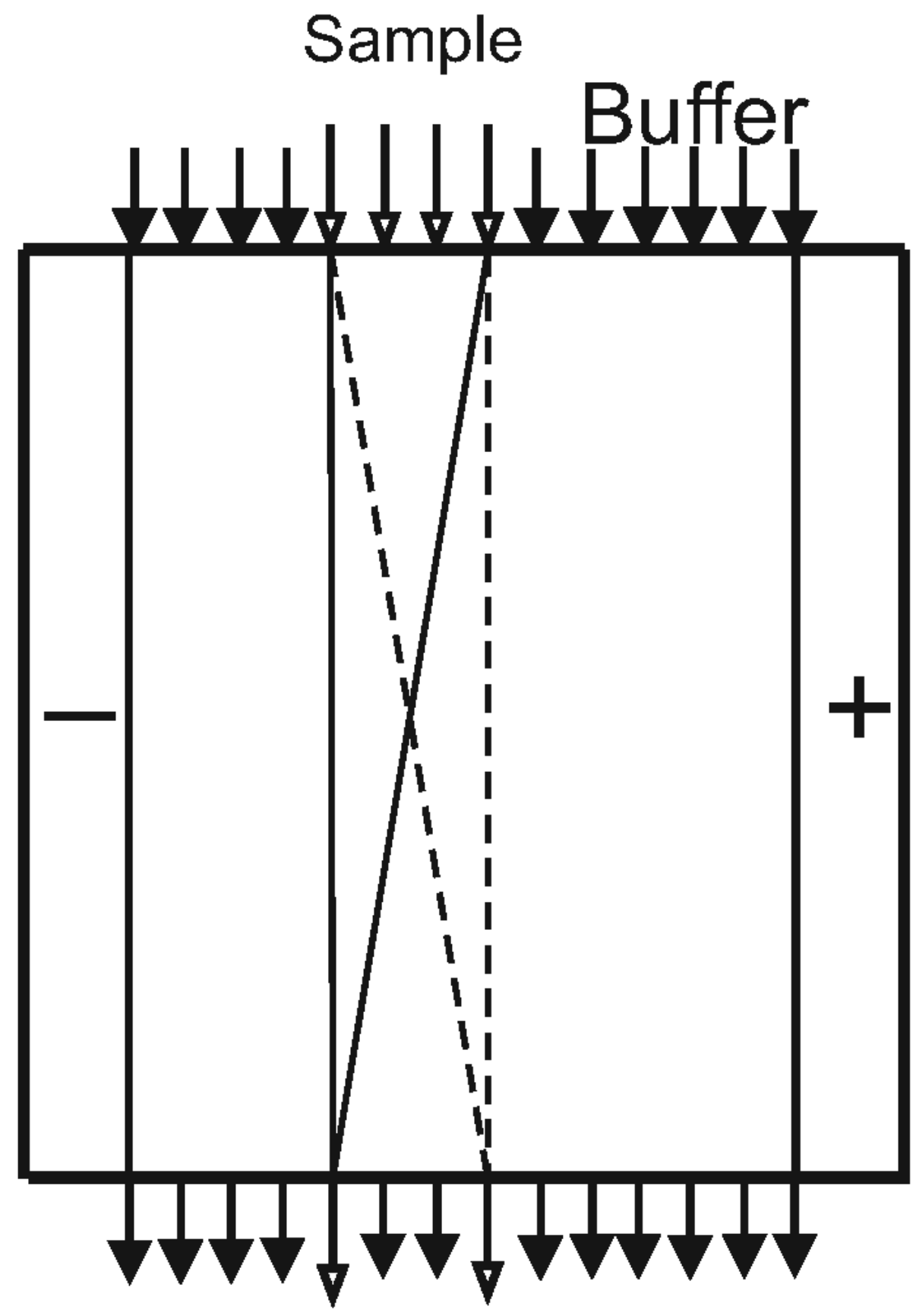

Figure 2

Operation of FFFSE. In the standard configuration, the two components in the mixture migrate in opposite directions and focus at the edges of the single electrical conductivity step. 


\section{$\mathrm{K}=9.0 \mathrm{mS} / \mathrm{cm}$}

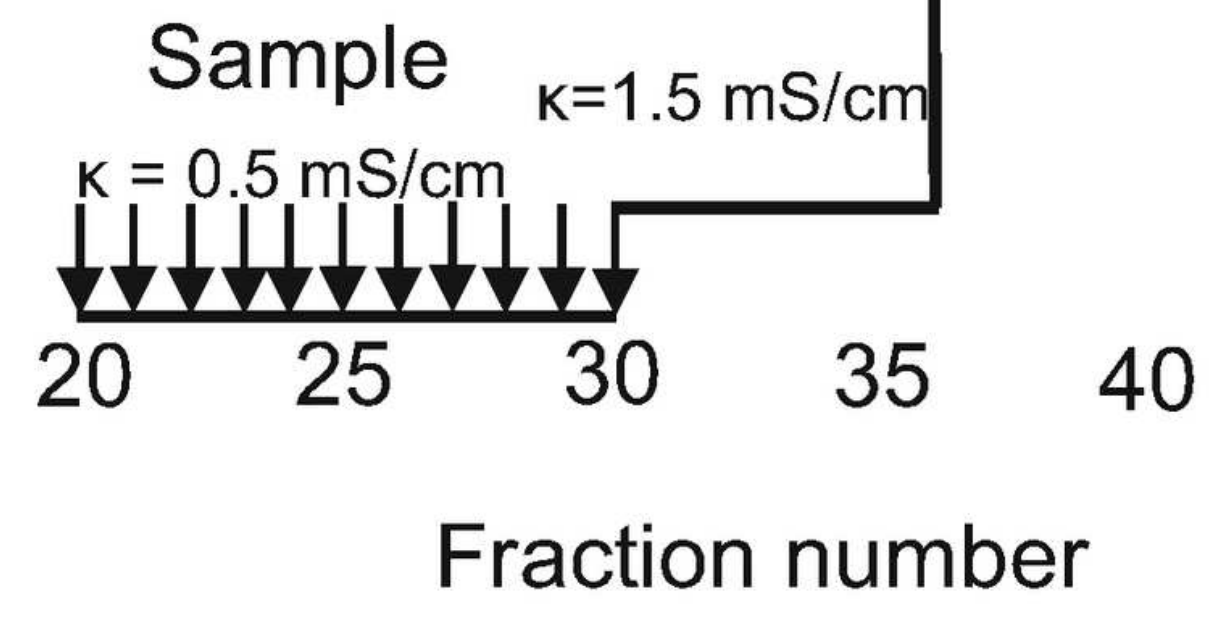

Figure 3

Modified configuration of FFFSE for separation of model proteins. The sample containing a mixture of proteins is injected in a broad zone between fractions 20 and 30 in the lowest electrical conductivity media, and two anodic conductivity steps are employed. The individual components of the mixture migrate very rapidly in the electrical field, and each component gets focused at the respective conductivity step immediately after fraction numbers 30 and 36 . 


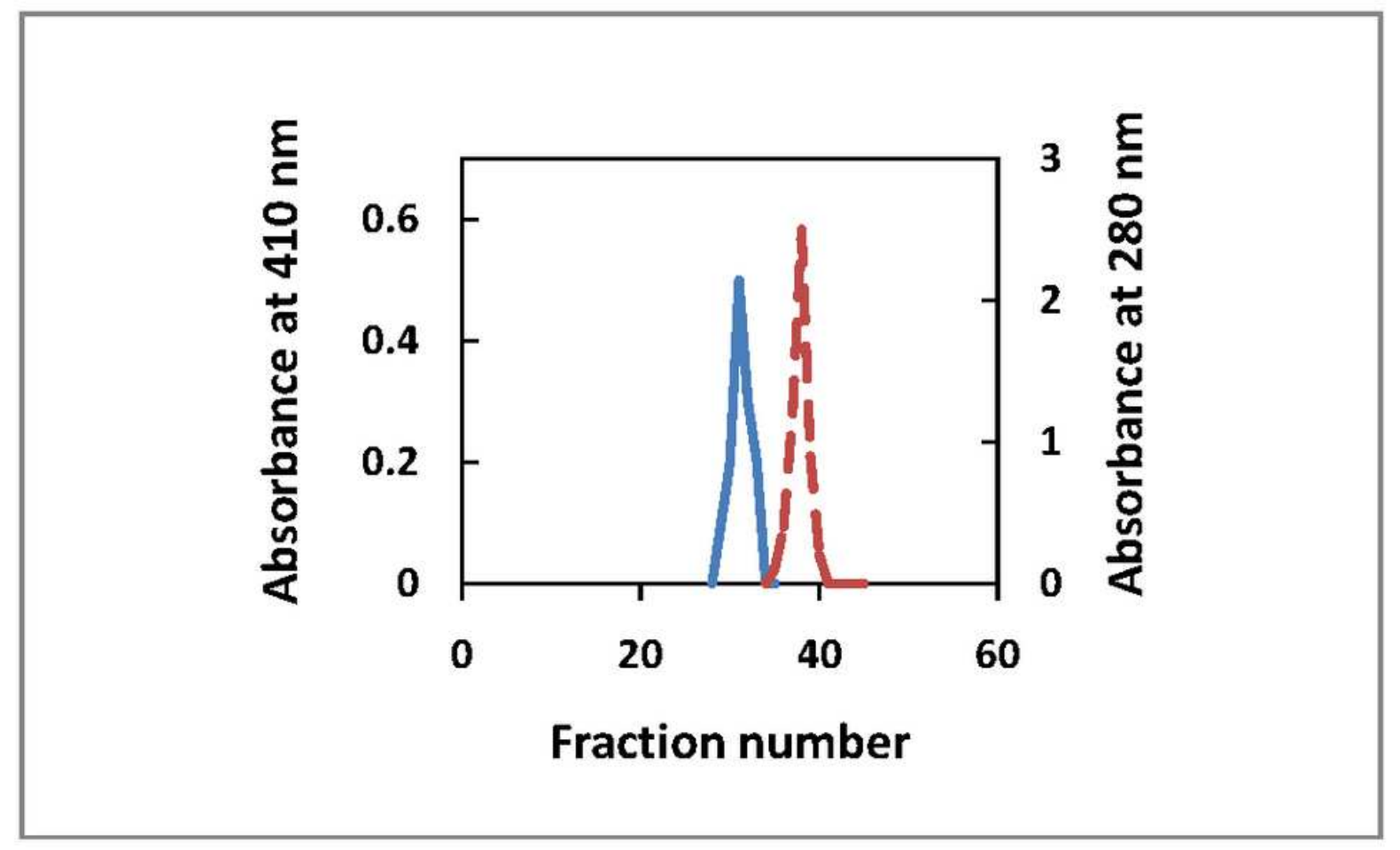

Figure 4

Separation of model proteins by continuous FFFSE with two anodic conductivity steps. Experimental conditions: Tris acetate buffer, $\mathrm{pH} 7.7, \mathrm{~K}=0.5,1.5,9.0 \mathrm{mS} / \mathrm{cm}$; buffer residence time $=5 \mathrm{~min}$, voltage $=$ $1.3 \mathrm{kV}$. The sample of the mixture of model proteins of $1 \mathrm{mg} / \mathrm{mL}$ concentration each after mixing was injected as a broad zone between fraction numbers 20 - 30. Bold line: Absorption at $410 \mathrm{~nm}$, Myoglobin. Dashed line: Absorption at $280 \mathrm{~nm}, \mathrm{BSA}$. The profile of electrical conductivity, $\mathrm{K}$ and the conductivity discontinuities across the separation chamber were as follows: Fractions $20-30, \mathrm{~K}=0.5 \mathrm{mS} / \mathrm{cm}$; fractions $30-36, \mathrm{k}=1.5 \mathrm{mS} / \mathrm{cm}$; fractions $36-40, \mathrm{k}=9.0 \mathrm{mS} / \mathrm{cm}$. 


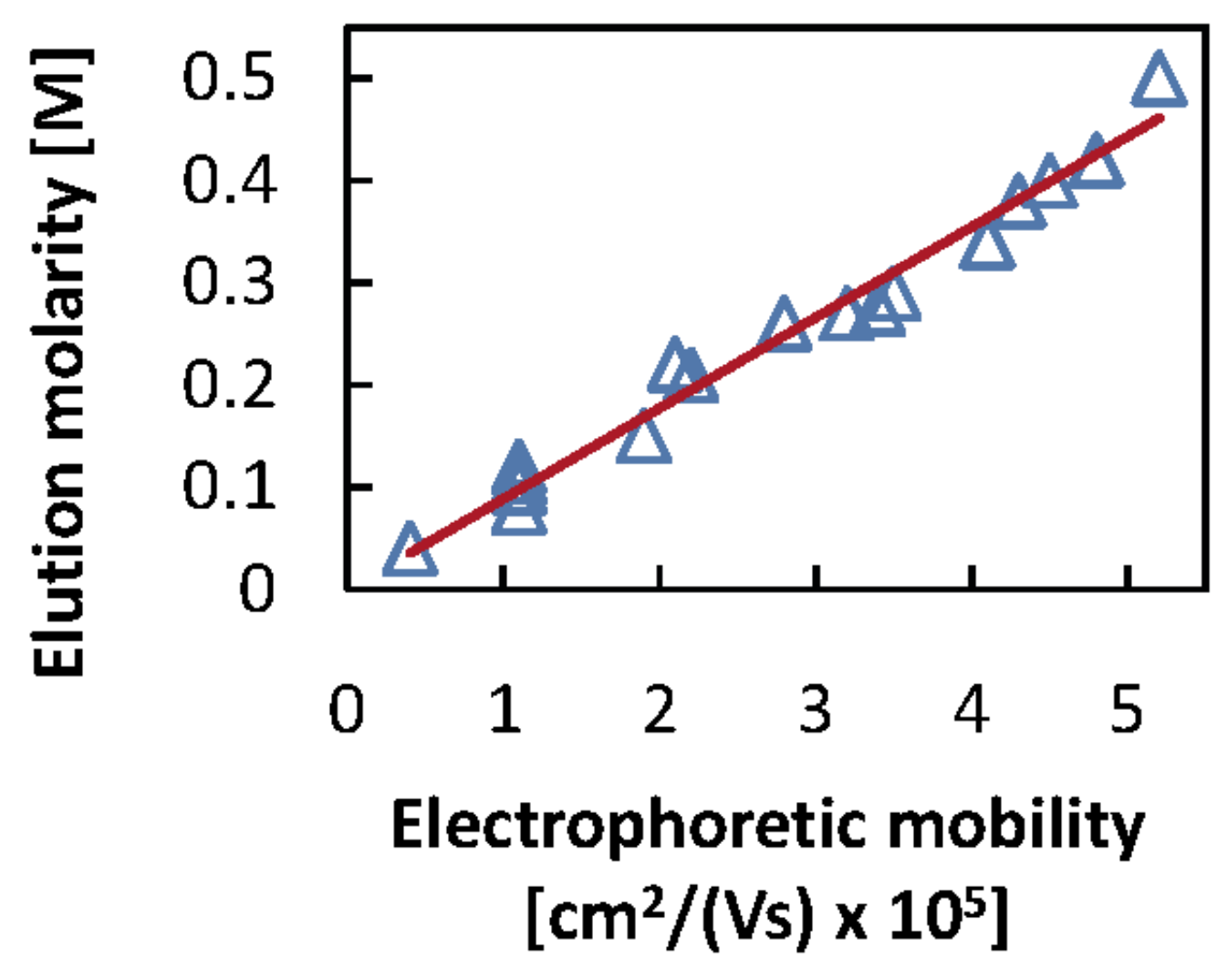

Figure 5

Correlation determined to help establish an electrokinetic basis for separation in FFZE/FFFSE for various proteins $(\mathrm{R} 2 \mathrm{=}$ 0.98). Tris acetate buffer, $\mathrm{pH}$ 7.7. The various proteins in ascending order of electrophoretic mobility are 1: myoglobin; 2: IgG; 3 : formate dehydrogenase from Candida boidinii; 4: catalase from bovine liver (sample 1); 5: D-Hic dehydrogenase; 6: catalase from bovine liver (sample 2); 7: formaldehyde dehydrogenase from Candida boidinii; 8: bovine serum albumin (BSA); 9: ovalbumin from chicken egg; 10: ferritin from horse spleen; 11: $\beta$-galactosidase from Escherichia coli; 12: bovine thyroglobulin; 13: methanol oxidase from Candida boidinii; 14: L-Hic dehydrogenase.

\section{Supplementary Files}

This is a list of supplementary files associated with this preprint. Click to download.

- GraphicalAbstractBioresourBioprocess.pdf 